\title{
Analysis of Logistic Growth Models
}

\author{
A.Tsoularis, \\ I.I.M.S., Massey University Albany Campus, Auckland, New Zealand \\ a.d.tsoularis@massey.ac.nz
}

\begin{abstract}
A variety of growth curves have been developed to model both unpredated, intraspecific population dynamics and more general biological growth. Most successful predictive models are shown to be based on extended forms of the classical Verhulst logistic growth equation. We further review and compare several such models and calculate and investigate properties of interest for these. We also identify and detail several previously unreported associated limitations and restrictions.

A generalized form of the logistic growth curve is introduced which is shown incorporate these models as special cases. The reported limitations of the generic growth model are shown to be addressed by this new model and similarities between this and the extended growth curves are identified. Several of its properties are also presented. We furthermore show that additional growth characteristics are accommodated by this new model, enabling previously unsupported, untypical population dynamics to be modelled by judicious choice of model parameter values alone.
\end{abstract}

\section{Introduction}

In order to model growth of biological systems numerous models have been introduced. These variously address population dynamics, either modelled discretely or, for large populations, mostly continuously. Others model actual physical growth of some property of interest for an organism or organisms.

The simple exponential growth model can provide an adequate approximation to such growth for the initial period. However, for populations, no predation or intraspecific competition is included. The population would therefore continue to increase unhindered (or inevitably reduce to zero if an initial growth reduction were present). Even in the case where predation was at most negligible, the model does not accommodate reductions due to intraspecific competition for environmental resources such as food and habitat. For the case of growth per se, unrestricted growth is also unrealistic. For example, as plants approach maturity, the physical characteristics of interest will reach a limiting dimension.

Verhulst [1] considered that, for the population model, a stable population would consequently have a saturation level characteristic: this is typically called the carrying capacity, $K$, and forms a numerical upper bound on the growth size. To incorporate this limiting form he introduced the logistic growth equation which is shown later to provide an extension to the exponential model. This logistic equation can also be seen to model physical growth provided $K$ is interpreted, rather naturally, as the limiting physical dimension. It is parameterized by the initial population size (or physical dimension), the initial growth rate, and $K$. For typical values of these, particularly, where the initial population size (or dimension) is smaller than $K$, the resulting logistic growth rate curve is sigmoidal. Furthemore, the point of inflection for the system is fixed such that the corresponding population (or dimension) is $\frac{K}{2}$. This places an undesirable restriction on the shape of the curve and clearly limits the generality of the model. We will see later that the Verhulst logistic growth model has formed the basis for several extended models. Each is a parameterised version of the original and provides a relaxation of this restriction.

Notwithstanding this limitation the logistic growth equation has been used to model many diverse biological systems. Carlson [2] reported the growth of yeast which is modelled well by the curve $[3,4]$. Morgan [5] ingeniously used the equation to describe herding behaviour of African 
elephants. Krebs [6] also used the Verhulst logistic equation to fit to population data for Peruvian anchovies.

There have been applications of the logistic model outside the field of Biology also. Fisher and Pry [7] have successfully exploited the logistic model to describe the market penetration of many new products and technologies. In this particular application of the logistic model $N$ represents a measure of the market already captured and $\frac{K-N}{K}$ that for the fraction of the market remaining to be captured. Marchetti and Nakicenovic [8] have given a summary of world energy usage and source substitution by employing the logistic model. Herman and Montroll [9] have shown that as basic an evolutionary process as the industrial revolution may also be modelled by logistic dynamics. Here, as the industrial revolution evolved, the fraction of the labour force in agriculture declined while the fraction in industry grew.

In this paper we present the major models of this form. We identify the population (or dimension) corresponding to the characteristic point of inflection for each and compare and contrast these. We also present previously unreported properties of these models. Finally, we introduce a generalized logistic equation which incorporates all these as special cases. We also investigate properties of this model.

\section{The Logistic Growth Curve}

The simplest realistic model of population dynamics is the one with exponential growth

with solution

$$
\frac{d N}{d t}=r N
$$

$$
N(t)=N_{0} e^{r t}
$$

where $r$ is the intrinsic growth rate and represents growth rate per capita. To remove unrestricted growth Verhulst [1] considered that a stable population would have a saturation level characteristic of the environment. To achieve this the exponential model was augmented by a multiplicative factor, $1-\frac{N}{K}$, which represents the fractional deficiency of the current size from the saturation level, $K$.

In Lotka's analysis [10] of the logistic growth concept the rate of population growth, $\frac{d N}{d t}$, at any moment $t$ is a function of the population size at that moment, $N(t)$, namely,

$$
\frac{d N}{d t}=f(N)
$$

Since a zero population has zero growth, $N=0$ is an algebraic root of the yet unknown function $f(N)$. By expanding $f(N)$ as a Taylor series near $N=0$ and setting $f(0)=0$ Lotka obtained the following power series

$$
\begin{aligned}
f(N) & =N f^{\prime}(0)+\frac{N^{2}}{2} f^{\prime \prime}(0) \\
& =N\left[f^{\prime}(0)+\frac{N}{2} f^{\prime \prime}(0)\right]
\end{aligned}
$$

where higher terms are assumed negligible.

By setting $f^{\prime}(0)=r$ and $f^{\prime \prime}(0)=-\frac{2 r}{K}$, where $r$ is the intrinsic growth rate of the population and $K$ is the carrying capacity, one is led to the Verhulst logistic equation 


$$
\frac{d N}{d t}=r N\left(1-\frac{N}{K}\right)
$$

The Verhulst logistic equation is also referred to in the literature as the Verhulst-Pearl equation after Verhulst, who first derived the curve, and Pearl [11], who used the curve to approximate population growth in the United States in 1920.

Equation (1) has solution

$$
N(t)=\frac{K N_{0}}{\left(K-N_{0}\right) e^{-r t}+N_{0}}
$$

where $N_{0}$ is the population size at time $t=0$.

The three key features of the logistic growth are:

(i) $\quad \lim _{t \rightarrow \infty} N(t)=K$, the population will ultimately reach its carrying capacity.

(ii) The relative growth rate, $\frac{1}{N} \frac{d N}{d t}$, declines linearly with increasing population size.

(iii) The population at the inflection point (where growth rate is maximum), $N_{\text {inf }}$, is exactly half the carrying capacity, $N_{i n f}=\frac{K}{2}$.

For $r>0$, the resulting growth curve has a sigmoidal shape and, from (2), is asymptotic to the carrying capacity. When $r<0$ and a reduction in the growth rate per capita is present, the growth curve is asymptotic to zero leading to population extinction. In the trivial case of no intrinsic growth rate, $r=0$, the population remains static at the initial value of $N_{0}$. Population biologists and ecologists are interested mainly in the case where $r>0$ and we restrict our investigations to this case in this paper.

Figure 1 depicts several logistic curves for various such values of $r$ with $N_{0}=10, K=100$. The larger the $r$ the faster in time the curve reaches the carrying capacity $K$. Figure 2 illustrates the fact that the population at the inflection point, $N_{i n f}=\frac{K}{2}=50$, regardless of the value assumed by the intrinsic growth rate, $r$.

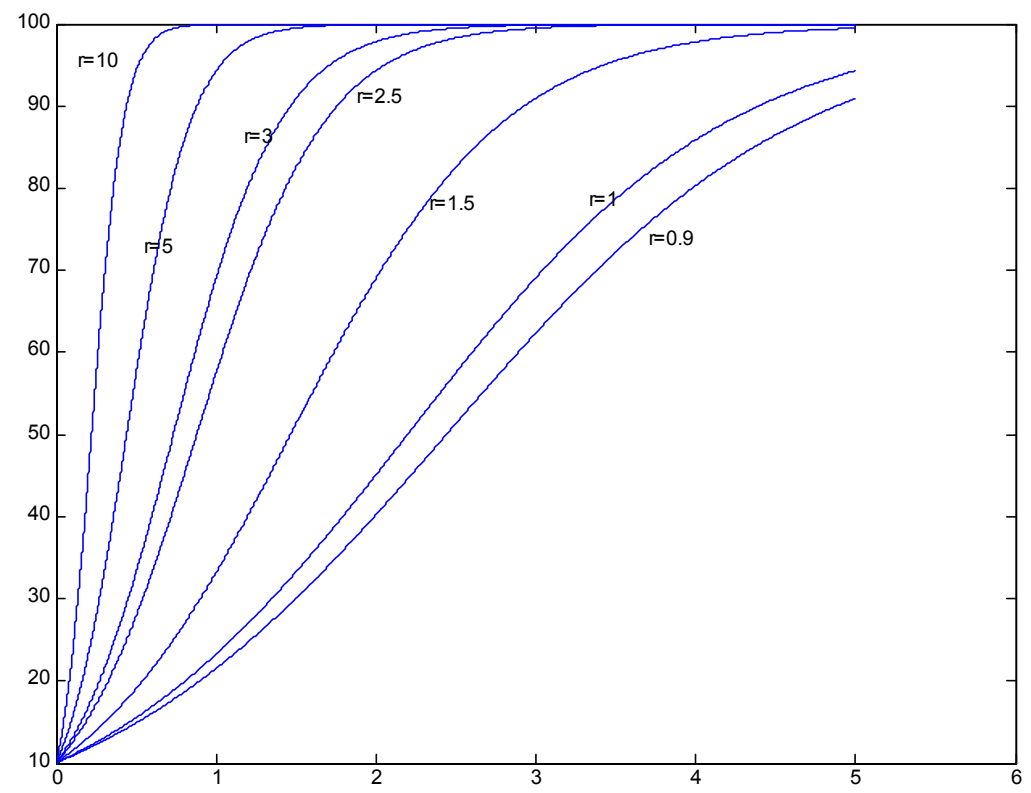


Figure 1. The evolution of population size in time for the Verhulst logistic growth.

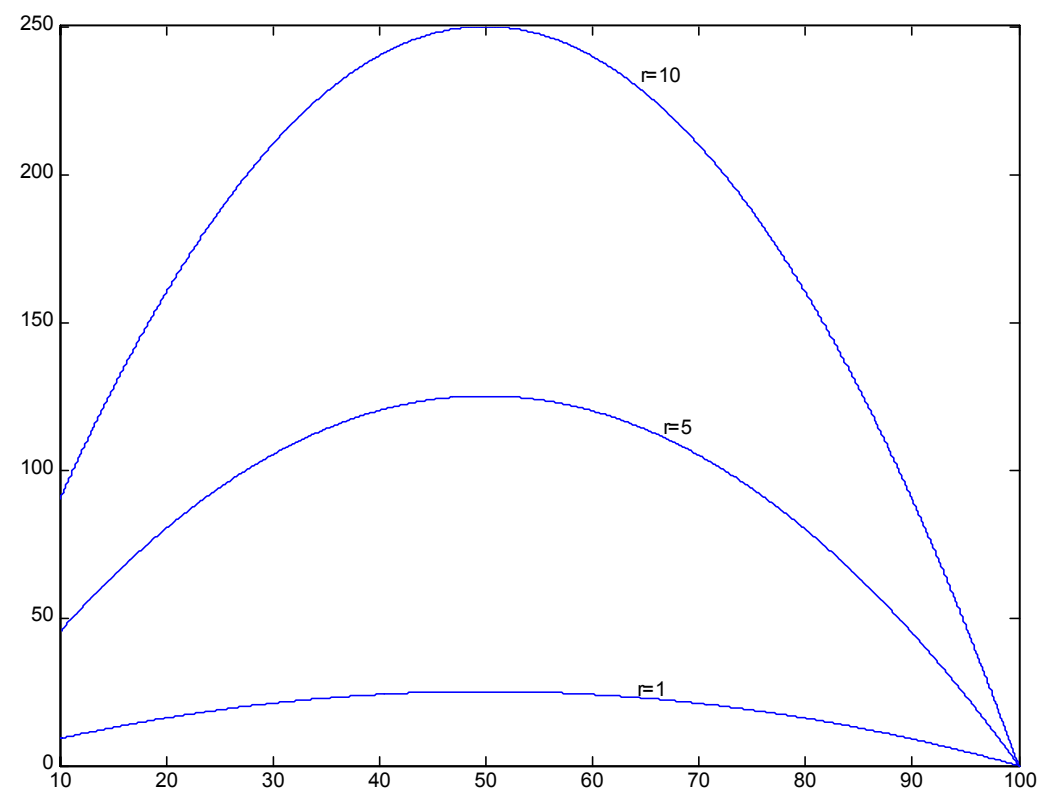

Figure 2. Plots of the growth rate versus population size for the Verhulst logistic growth.

\section{Extended Logistic Growth Models}

Since the original work of Verhulst [1] and Pearl and Reed [11] there have been several contributions suggesting alternative functional forms, $f(N)$, for growth whilst retaining the sigmoid and asymptotic property of the Verhulst logistic curve. In the plant sciences, Richards [12] was the first to apply a growth equation developed first by Von Bertalanffy [13] to describe the growth of animals. Richards growth curve was used for fitting experimental data by Nelder [14], who used the term generalized logistic equation to describe the equation. Blumberg [15] introduced the hyperlogistic equation as a generalization of Richards' equation. Turner and coauthors [16,17] suggested a further generalization of the logistic growth and termed their equation the generic logistic equation. In a more recent survey paper Buis [18] revisited the previous works on logistic growth functions and outlined some of their respective properties.

In this section we derive several well known growth functions which extend the standard Verhulst equation. In addition, we examine the presence or absence of the sigmoid feature that characterizes most growth curves and is responsible for the existence of an inflection point.

\subsection{Generic Growth Function}

Turner and co-authors [17] proposed a modified Verhulst logistic equation which they termed the generic growth function. This has the form

$$
\frac{d N}{d t}=r N^{1+\beta(1-\gamma)}\left[1-\left(\frac{N}{K}\right)^{\beta}\right]^{\gamma}
$$

where $\beta, \gamma$ are positive exponents and $\gamma<1+\frac{1}{\beta}$. This has the solution, 


$$
N(t)=\frac{K}{\left[1+\left[(\gamma-1) \beta r K^{\beta(1-\gamma)} t+\left[\left(\frac{K}{N_{0}}\right)^{\beta}-1\right]^{1-\gamma}\right]^{\frac{1}{1-\gamma}}\right]^{\frac{1}{\beta}}}
$$

The population at the inflection point, $N_{\text {inf }}$, is given by

$$
N_{\text {inf }}=\left(1-\frac{\beta \gamma}{1+\beta}\right)^{\frac{1}{\beta}} K
$$

For $\beta=\gamma=1$ the functional form for $N_{\text {inf }}$ reduces to that for the Verhulst logistic equation. The condition $\quad \gamma<1+\frac{1}{\beta}$ ensures that $N_{\text {inf }}>0$. For extreme values of $\beta$ and $\gamma$ we obtain the following limits for $N_{\text {inf }}$ :

$$
\begin{aligned}
& \lim _{\beta \rightarrow 0} N_{i n f}=K e^{-\gamma}, \quad 0<\gamma<\infty \\
& \lim _{\beta \rightarrow \infty} N_{i n f}=K, \quad 0<\gamma<1 \\
& \lim _{\gamma \rightarrow 0} N_{i n f}=K, \quad 0<\beta<\infty \\
& \lim _{\gamma \rightarrow \infty} N_{i n f}=0, \quad \beta \rightarrow 0
\end{aligned}
$$

Figure 3 displays several generic growth curves evolving in time $t$ and figure 4 presents the growth rate versus time evolution for $N_{0}=10, K=100$. A visible inflection point occurs for $\beta=$ 5.0, $\gamma=1.0$, given by $N_{\text {inf }}=\left(\frac{1}{6}\right)^{0.2} K \approx 70$, and is clearly seen in Figure 4 . For $\beta=0.5, \gamma=$ 2.5, $N_{\text {inf }}=\left(\frac{1}{6}\right)^{2} K \approx 2.5<10$, and no inflection is present. Also no inflection occurs for $\beta=$ 0.2, $\gamma=5.0, N_{\text {inf }}=\left(\frac{1}{6}\right)^{5} K \approx 0$. An inflection point, $N_{\text {inf }}=25$, is present for $\beta=1.0, \gamma=1.5$. More symmetric graphs with inflection occurring at around half the value of $K$, are those with $\beta$ $=2.0, \gamma=1.0$ and $\beta=3.0, \gamma=1.0$, giving $N_{\text {inf }} \approx 58$ and $N_{\text {inf }} \approx 63$ respectively. 


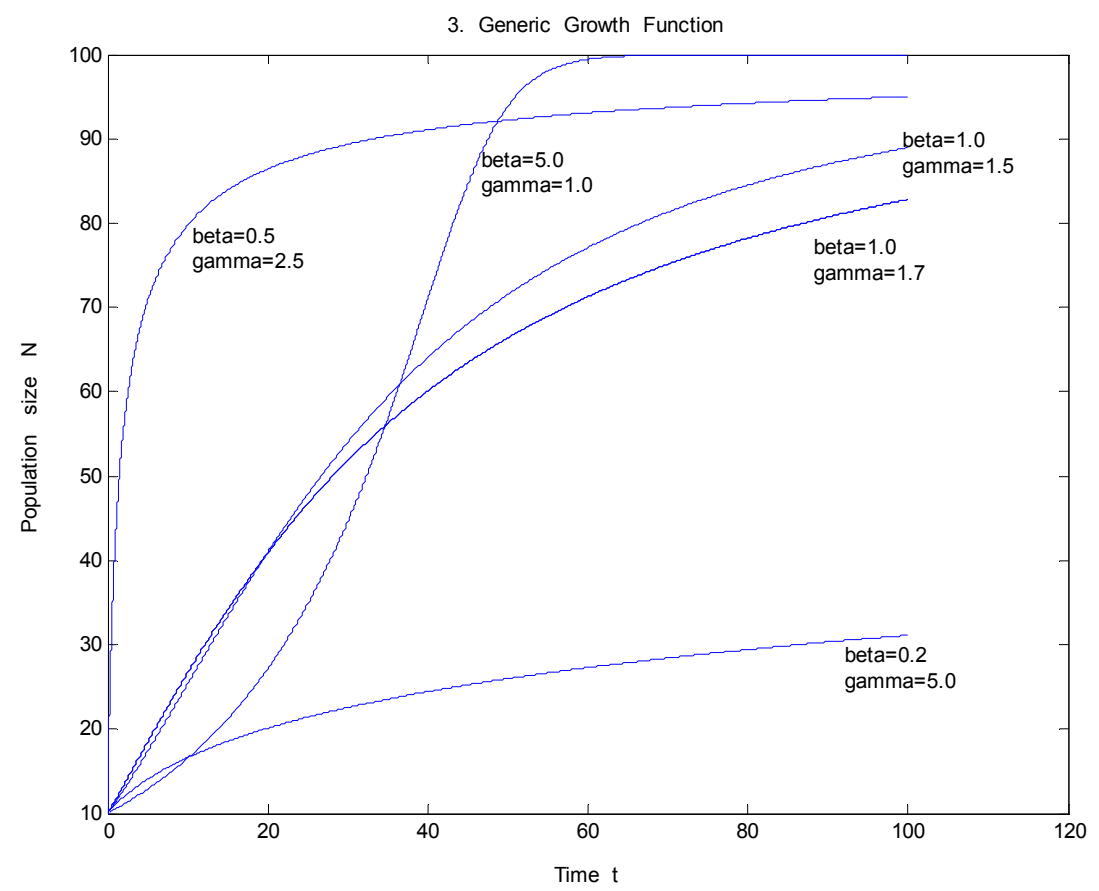

Figure 3. The evolution of population size in time for several parameter pairs $(\beta, \gamma)$ according to the generic growth form.

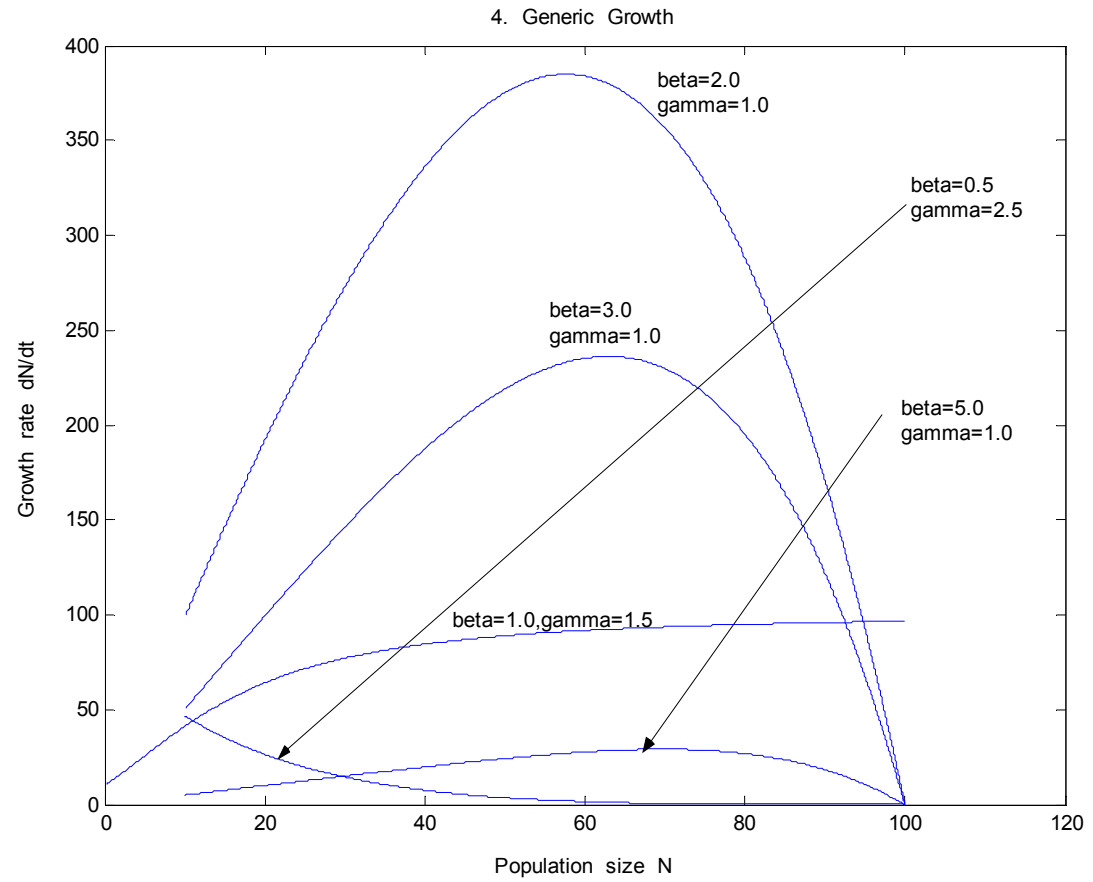

Figure 4. Growth rate versus size plots for the generic growth function.

\subsection{Blumberg's equation}


Blumberg [15] introduced another growth equation based on a modification of the Verhulst logistic growth equation to model population dynamics or organ size evolution. Blumberg observed that the major limitation of the logistic curve was the inflexibility of the inflection point. He further observed that attempts to modify the constant intrinsic growth rate term, $r$, treating this as a time-dependent polynomial to overcome this limitation, often leads to underestimation of future values (see also [10]). Blumberg therefore introduced what he called the hyperlogistic function, accordingly

$$
\frac{d N}{d t}=r N^{\alpha}\left(1-\frac{N}{K}\right)^{\gamma}
$$

Blumberg's equation is consistent with the Turner and co-authors' generic equation (3) when $\alpha=$ $2-\gamma, \beta=1$, and $\gamma<2$.

Equation (5) can be re-formulated as the integral equation

$$
\int_{\frac{N_{0}}{K}}^{\frac{N(t)}{K}} x^{-\alpha}(1-x)^{-\gamma} d x=r K^{\alpha-1} t
$$

This does not always afford a closed form analytical solution. Blumberg therefore catalogued analytic expressions (when an explicit integration can be carried out) of the growth function $N(t)$ for various values of the parameters $\alpha$ and $\gamma$.

The population at the inflection point, $N_{\text {inf }}$, is given by

$$
N_{\text {inf }}=\frac{\alpha}{\alpha+\gamma} K
$$

This also coincides with that of the Verhulst logistic equation when $\alpha=\gamma$. For $\alpha>\gamma$ the inflection occurs very near the carrying capacity, and for $\alpha<<\gamma, \quad N_{\text {inf }}$ approaches 0 and inflection occurs only if $N_{0}<N_{\text {inf }}$.

Figure 5 and figure 6 exhibit respectively the population size as a function of time and the growth rate variation with population size for several values of the parameters $\alpha$ and $\gamma$. In Figure 6 the gradual transition of the inflection point from values less than $\frac{K}{2}=50$, when $\alpha<\gamma$, to values greater than $\frac{K}{2}$, when $\alpha>\gamma$, can be clearly seen. 


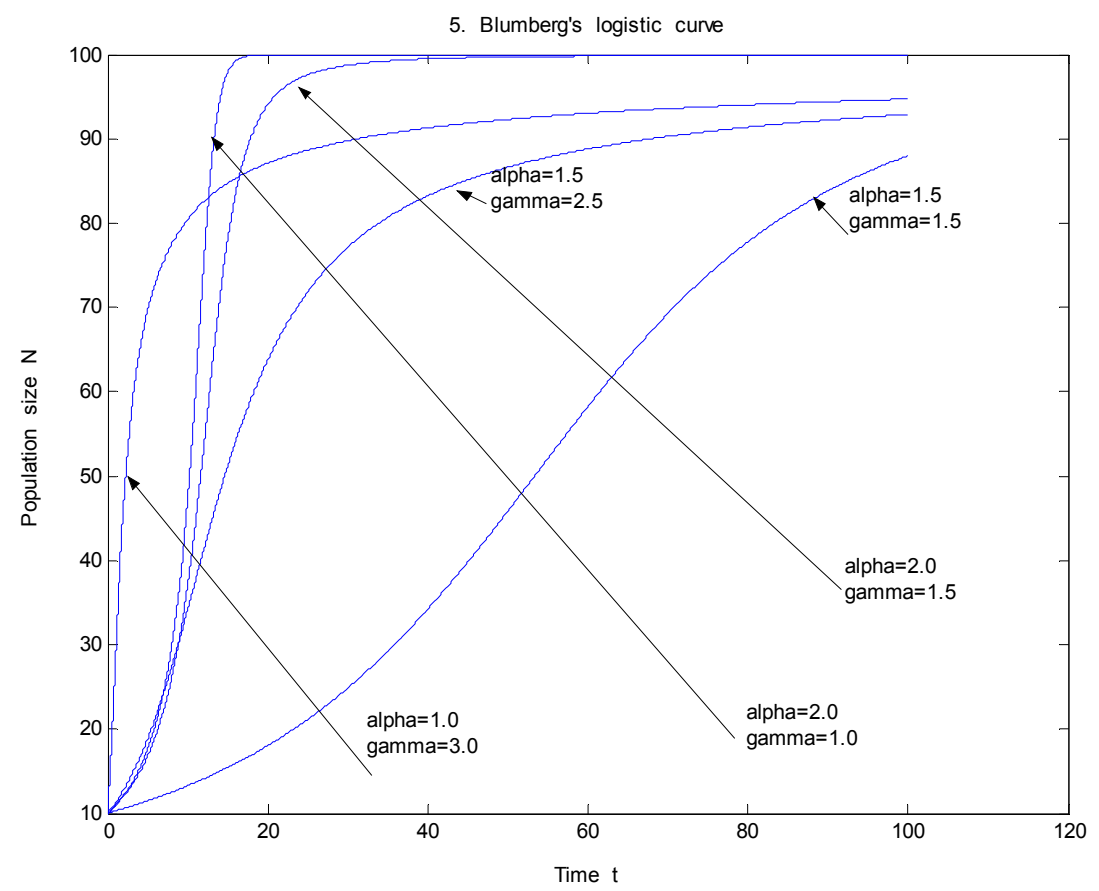

Figure 5. Population size growth versus time according to Blumberg's functional form.

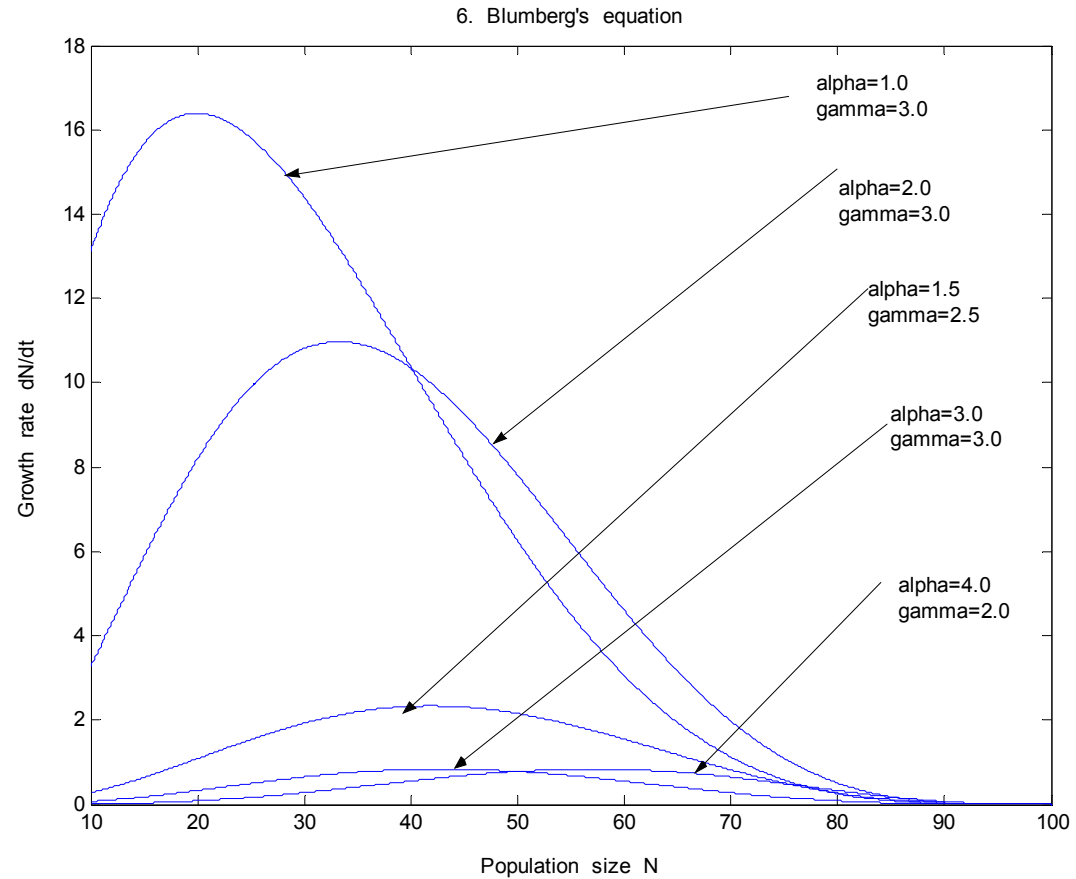

Figure 6. Growth rate versus size graph for Blumberg's equation. The movement of the inflection point from the left, where $\alpha<\gamma$, to the right, where $\alpha>\gamma$, is clearly visible

\subsection{Von Bertalanffy's growth equation}

Von Bertalanffy [13] introduced his growth equation to model fish weight growth. Here the Verhulst logistic growth curve was modified to accommodate crude "metabolic types" based upon physiological reasoning. He proposed the form given below which can be seen to be a special case of the Bernoulli differential equation: 


$$
\frac{d N}{d t}=r N^{\frac{2}{3}}\left[1-\left(\frac{N}{K}\right)^{\frac{1}{3}}\right]
$$

which has solution

$$
N(t)=K\left[1+\left[1-\left(\frac{N_{0}}{K}\right)^{\frac{1}{3}}\right] e^{-\frac{1}{3} r K^{-\frac{1}{3}} t}\right]^{3}
$$

The Bertalanffy model cannot be derived from the Turner model as the values of the exponents, $\alpha=\frac{2}{3}, \beta=\frac{1}{3}, \gamma=1$, violate the condition $\alpha=1+\beta(1-\gamma)$ stipulated by Turner et al. (see Section 3.5). It cannot therefore be seen as a special case and should be viewed as a separate model accordingly.

Here, $N_{\text {inf }}$ is given by

$$
N_{i n f}=\left(\frac{\frac{2}{3}}{\frac{2}{3}+\frac{1}{3}}\right)^{3} K=\frac{8}{27} K
$$

which, whilst differing from that for the Verhulst curve, still represents a substantial restriction for general modelling purposes.

Figures 7 and 8 display respectively a typical Von Bertalanffy weight growth curve and its inflection.

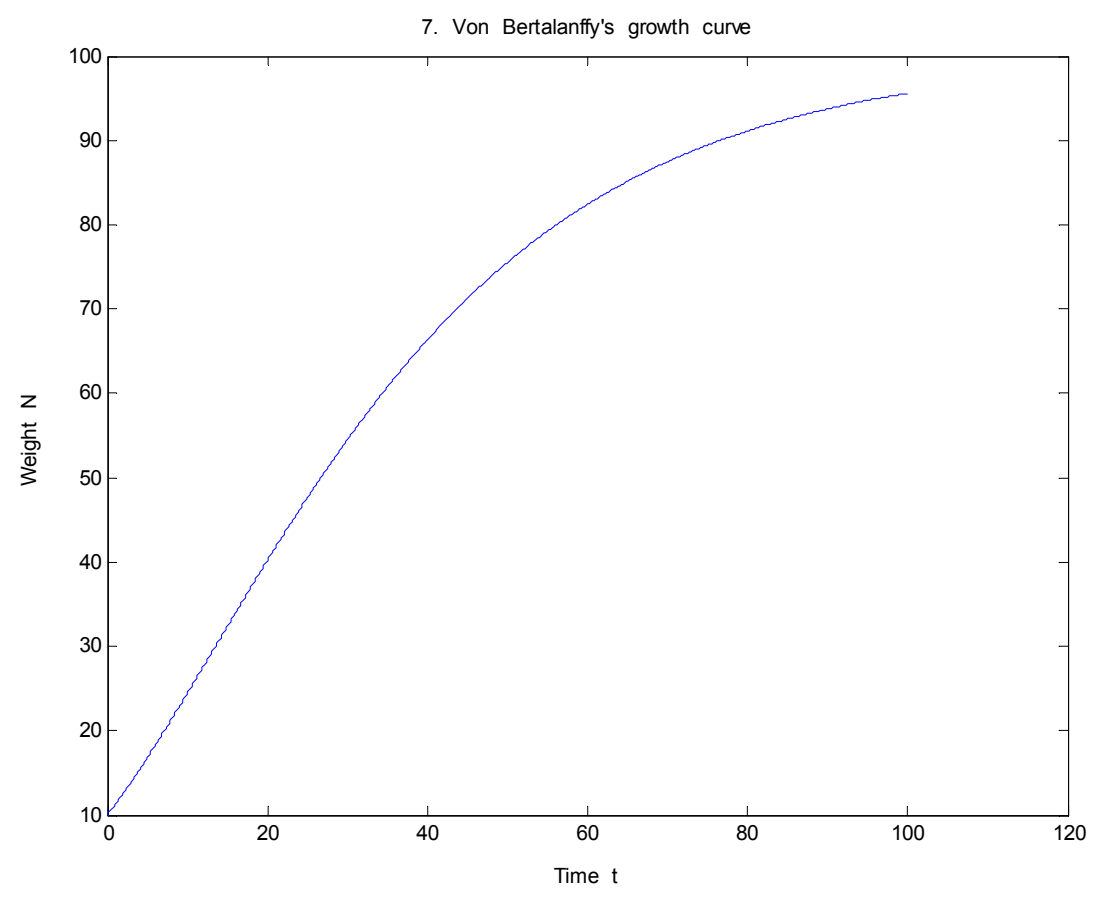

Figure 7. Von Bertalanffy's weight growth curve. 


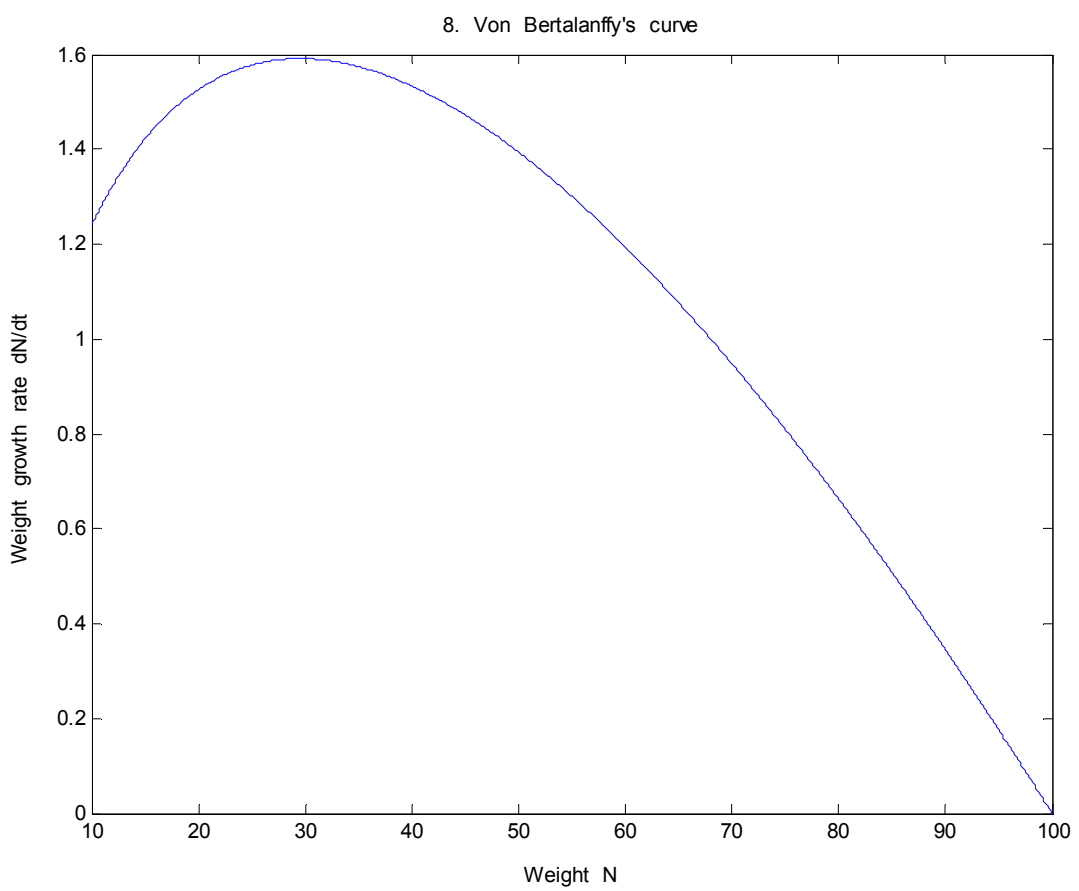

Figure 8. The growth rate for Von Bertalanffy's form realizes its maximum at around 30, when the asymptotic value is $K=100$.

\subsection{Richards growth equation}

Richards extended the growth equation developed by Von Bertalanffy to fit empirical plant data [12]. Richards's suggestion was to use the following equation which is also a special case of the Bernoulli differential equation

$$
\frac{d N}{d t}=r N\left[1-\left(\frac{N}{K}\right)^{\beta}\right]
$$

which has the solution

$$
N(t)=K\left[1-e^{-\beta r t}\left[1-\left(\frac{N_{0}}{K}\right)^{-\beta}\right]\right]^{-\frac{1}{\beta}}
$$

Unlike its Von Bertalanffy antecedent however, the Richards growth function does follow from the Turner model (Section 3.5) in the case where, $\gamma=1$.

Here inflection occurs at

$$
N_{\text {inf }}=\left(\frac{1}{1+\beta}\right)^{\frac{1}{\beta}} K
$$

For $\beta=1$, (6) trivially reduces to the Verhulst logistic growth equation (1). For extreme values of $\beta$ we obtain the following values for $N_{\text {inf }}$ :

$$
\begin{gathered}
\lim _{\beta \rightarrow 0} N_{i n f}=K e^{-1} \\
\lim _{\beta \rightarrow \infty} N_{i n f}=K
\end{gathered}
$$

Consistent with the previous observation, the above values also follow from the corresponding population value at the inflection point for the generic growth function with $\gamma=1$. 
Figure 9 illustrates four different Richards growth curves with $\beta=0.01,0.05,3.0$ and 6.0. Figure 10 displays the variation of the weight growth rate for small values for $\beta, \beta=0.01$ and $\beta=0.05$ giving inflection at $N_{\text {inf }} \approx 36$ and $N_{\text {inf }} \approx 38$ (both values are approximately equal to $\frac{K}{e}$ ), and larger $\beta$ values resulting in higher inflection values.

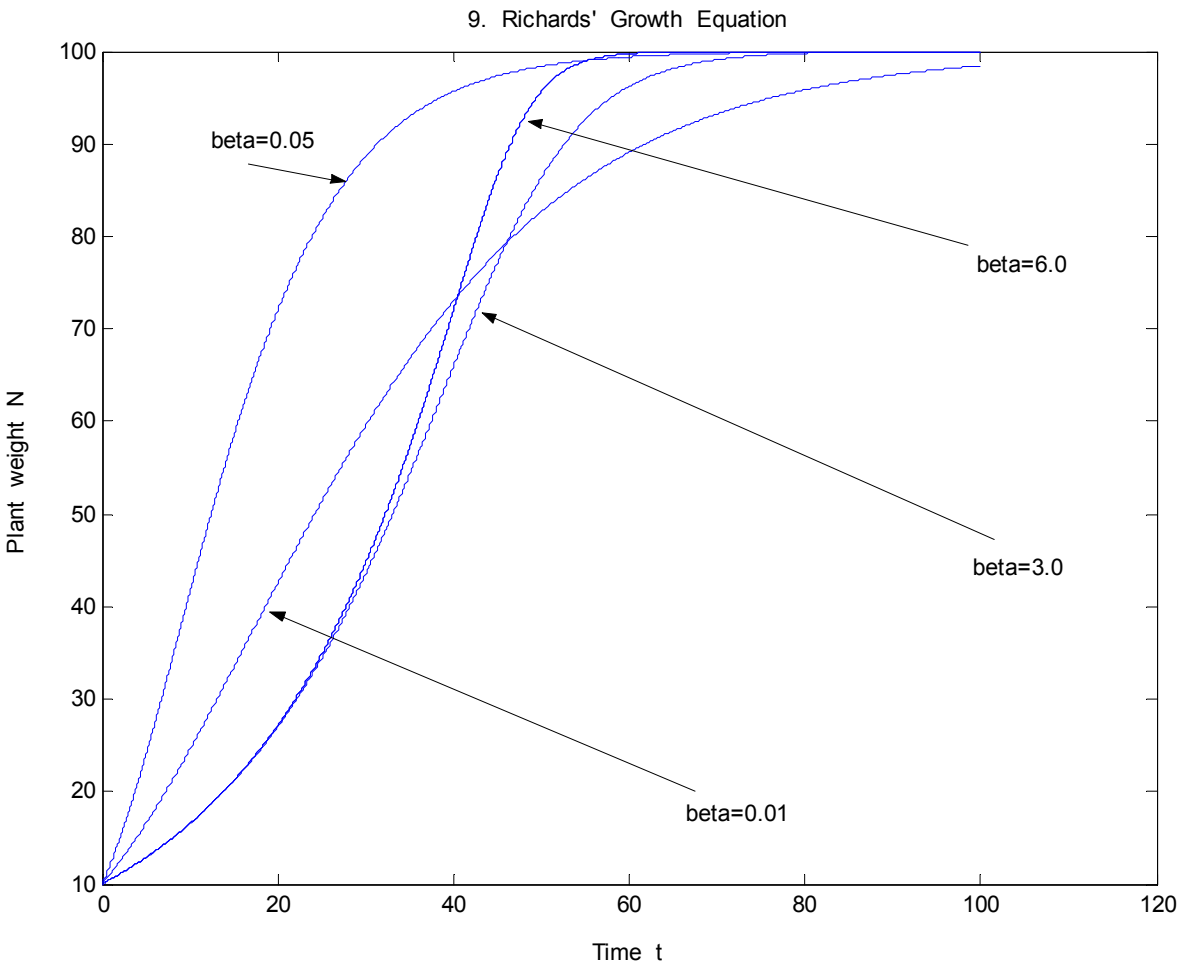

Figure 9. Plant weight growth in time according to Richards' equation. 


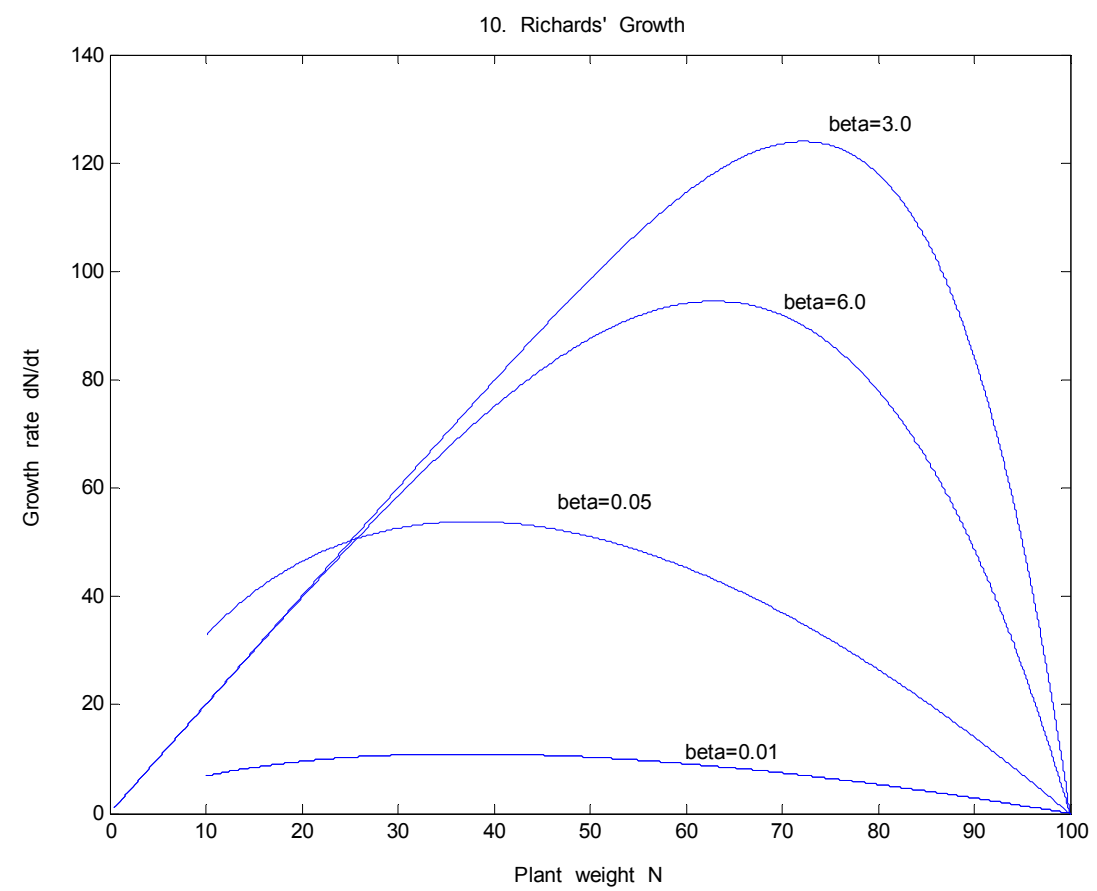

Figure 10. Weight growth rate versus weight plot for Richards' equation.

\subsection{Gompertz growth function}

The Gompertz growth curve can be derived from the following form of the logistic equation as a limiting case:

$$
\begin{aligned}
\frac{d N}{d t} & =\frac{r}{\beta^{\gamma}} N\left[1-\left(\frac{N}{K}\right)^{\beta}\right]^{\gamma} \\
& =\frac{r N}{K^{\beta \gamma}}\left(\frac{K^{\beta}-N^{\beta}}{\beta}\right)^{\gamma} \\
& =r^{\prime} N\left(\frac{K^{\beta}-N^{\beta}}{\beta}\right)^{\gamma}
\end{aligned}
$$

where $r^{\prime}=\frac{r}{K^{\beta \gamma}}$.

Looking at $\frac{K^{\beta}-N^{\beta}}{\beta}$ in the limit as $\beta \rightarrow 0$ we obtain 


$$
\begin{aligned}
& \lim _{\beta \rightarrow 0} \frac{K^{\beta}-N^{\beta}}{\beta}=\lim _{\beta \rightarrow 0} \frac{e^{\beta \ln K}-e^{\beta \ln N}}{\beta} \\
& =\lim _{\beta \rightarrow 0} \frac{\sum_{n=0}^{\infty} \frac{(\beta \ln K)^{n}}{n !}-\sum_{n=0}^{\infty} \frac{(\beta \ln N)^{n}}{n !}}{\beta} \\
& =\ln \left(\frac{K}{N}\right)+\lim _{\beta \rightarrow 0} \sum_{n=2}^{\infty} \frac{\beta^{n-1}}{n !}\left[(\ln K)^{n}-(\ln N)^{n}\right] \\
& =\ln \left(\frac{K}{N}\right)
\end{aligned}
$$

Similarly, $\lim _{\beta \rightarrow 0}\left(r^{\prime}\right)=r, \gamma>0$.

The growth rate modelled by the Gompertz function is given by

$$
\frac{d N}{d t}=r N\left[\ln \left(\frac{K}{N}\right)\right]^{\gamma}
$$

With $\gamma>0, \gamma \neq 1$, this special case is more usually known as the hyper-Gompertz (Turner et al. [17]), generalized ecological growth function, or simply generalized Gompertz function. Equation (7) can be conveniently rewritten as follows

$$
\frac{d}{d t}\left[\ln \left(\frac{N}{K}\right)\right]=r(-1)^{\gamma}\left[\ln \left(\frac{N}{K}\right)\right]^{\gamma}
$$

which upon integration leads to the analytic solution

$$
N(t)=K \exp \left\{\left[\ln \left(\frac{N_{0}}{K}\right)\right]^{1-\gamma}+r^{\prime}(-1)^{\gamma}(1-\gamma) t\right\}^{\frac{1}{1-\gamma}}
$$

The population at the inflection point, $N_{\text {inf }}$, is obtained by differentiating both sides of $\frac{d N}{d t}=r^{\prime} N\left(\frac{K^{\beta}-N^{\beta}}{\beta}\right)^{\gamma}$, setting $\frac{d^{2} N}{d t^{2}}=0$, and subsequently taking the limit:

$$
N_{\text {inf }}=\lim _{\beta \rightarrow 0}\left(\frac{1}{1+\beta \gamma}\right)^{\frac{1}{\beta}} K=K e^{-\gamma}
$$

The above form can also be obtained by differentiating both sides of equation (7) and setting $\frac{d^{2} N}{d t^{2}}=0$.

For relatively large increasing positive values of $\gamma$ the inflection point tends to 0 . Indeed, to achieve an inflection point, it is necessary that $K e^{-\gamma}>N_{0}$, whereas for increasingly small values of $\gamma$, the inflection point tends to $K$.

For $\gamma=1$ the equation

$$
\frac{d N}{d t}=r N\left[\ln \left(\frac{K}{N}\right)\right]
$$

is the ordinary Gompertz growth (see [19], [20]). The solution to (8) is 


$$
N(t)=K \exp \left\{\ln \left(\frac{N_{0}}{K}\right) e^{-r t}\right\}
$$

The population value at the inflection point, $N_{i n f}=K e^{-1}$, is obtained from that for the generalized Gompertz growth function with $\gamma=1$.

Figure 11 depicts three hyper-Gompertz growth curves $(\gamma>0, \gamma \neq 1)$ and the ordinary Gompertz growth $(\gamma=1)$ for $N_{0}=10$ and $K=100$. As predicated, relatively large values of $\gamma$ result in an inflection near the origin, $\gamma=3$ gives $N_{\text {inf }} \approx 4.5<N_{0}, \gamma=2.5$ gives $N_{\text {inf }} \approx 7.5<N_{0}$, whereas relatively low values of $\gamma$ result in a visible inflection point. A value of $\gamma=0.5$ gives $N_{\text {inf }} \approx 60$, and $\gamma=1$ gives $N_{\text {inf }} \approx 35.5$, as shown in Figure 12 .

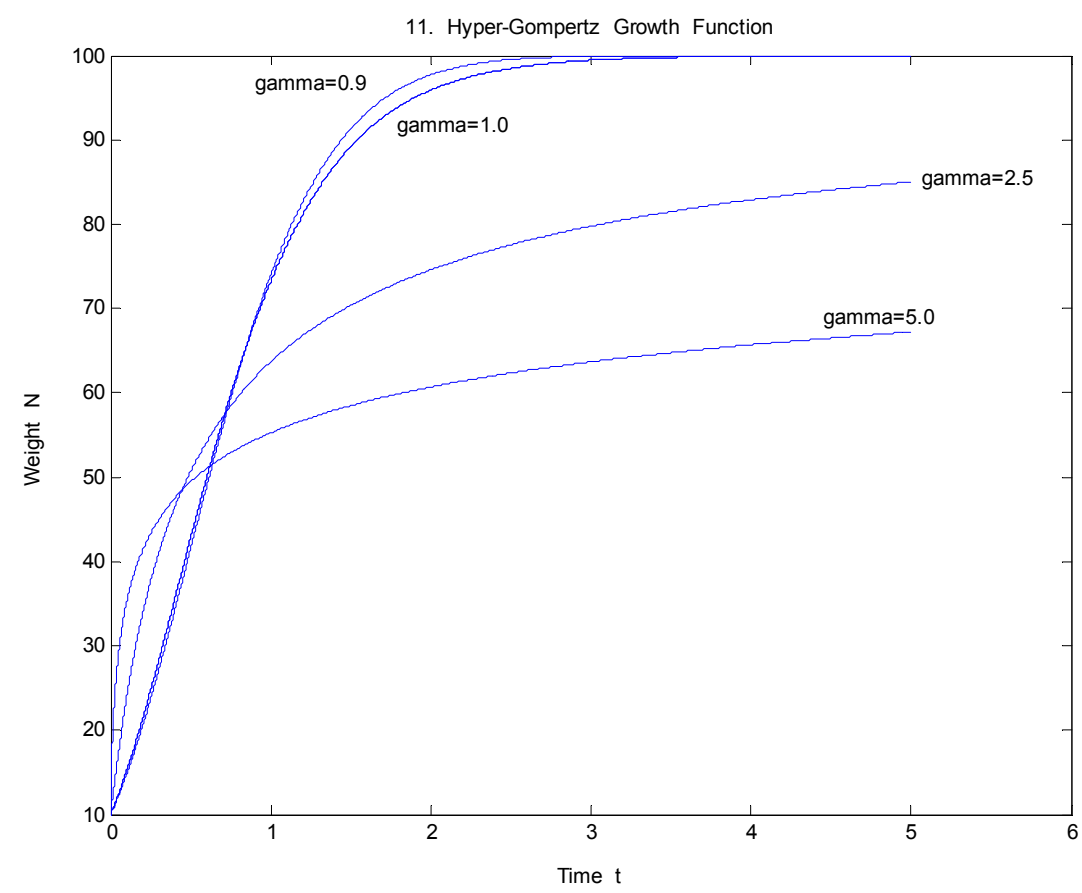

Figure 11. Hyper-Gompertz and ordinary Gompertz $(\gamma=1)$ growth plots. 


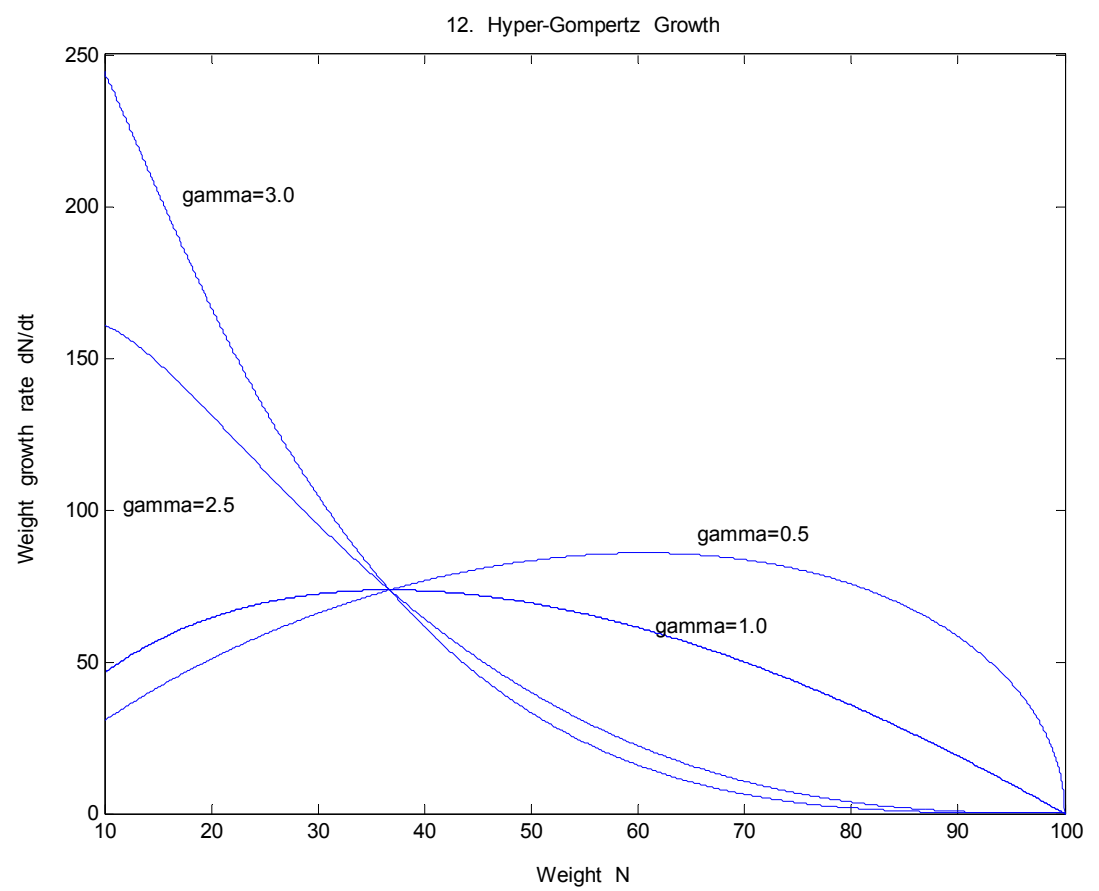

Figure 12. Hyper-Gompertz and ordinary Gompertz $(\gamma=1)$ weight growth rates versus weight curves.

\section{Generalized Logistic growth function}

Here we propose a generalized logistic growth equation which incorporates all previously reported functional forms as special cases. We will adopt the term generalized logistic equation in our exposition, a term first used by Nelder [14] to describe the Richards equation. We believe the adopted term is an appropriate one as it connotes exactly what it purports to achieve.

\subsection{Definition and properties of the generalized logistic function}

We define the generalized logistic function thus

$$
\frac{d N}{d t}=r N^{\alpha}\left[1-\left(\frac{N}{K}\right)^{\beta}\right]^{\gamma}
$$

where $\alpha, \beta, \gamma$ are positive real numbers. In this paper we confine ourselves to positive values for these parameters and that for $r$, as negative exponents do not always provide a biologically plausible model. Unlike Lotka's derivation of the Verhulst logistic growth equation from the truncation of the Taylor series expansion of $f(N)$ near $N=0$, (9) cannot be derived from such an expansion unless $\alpha, \beta, \gamma$ are all positive integers, in which case a power series can be generated with the first $\alpha$ terms $f(0)=f^{\prime}(0)=f^{\prime \prime}(0)=\cdots=f^{(\alpha-1)}(0)=0$.

By differentiating (9) and setting the second derivative to zero, we obtain the following parametric expression for the population value, $N_{\text {inf }}$, at the inflection point

$$
N_{\text {inf }}=\left(1+\frac{\beta \gamma}{\alpha}\right)^{-\frac{1}{\beta}} K
$$

Clearly if $N_{i n f}<N_{0}$, no inflection is possible as the population will have started with this initial value, $N_{0}$, and with a positive intrinsic growth per capita rate thus ensuring that $N_{\text {inf }}$ is not achievable. 
The above expression can be seen to contain the inflection points of all previous curves as special cases. For example, with $\alpha=1+\beta(1-\gamma)$, it reduces to the inflection value for the generic growth model given by (4).

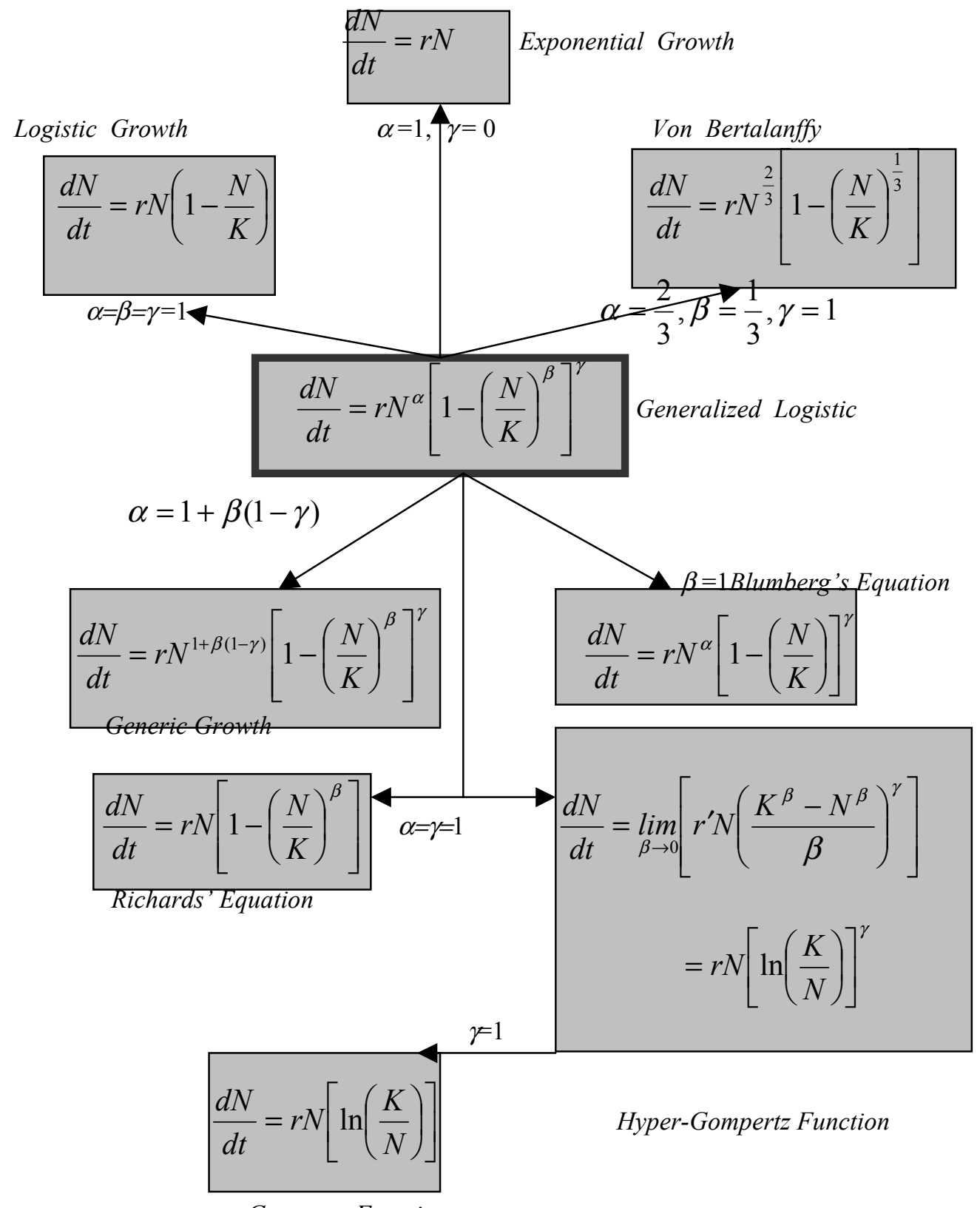

Gompertz Function

Figure 13. The generalized logistic curve and its derivative models.

Figure 14 is a display of the generalized logistic growth curve with parameters $\alpha, \beta, \gamma$ chosen at random (but kept positive), and figure 15 shows the growth rates with their respective maxima for the same parameter range. 


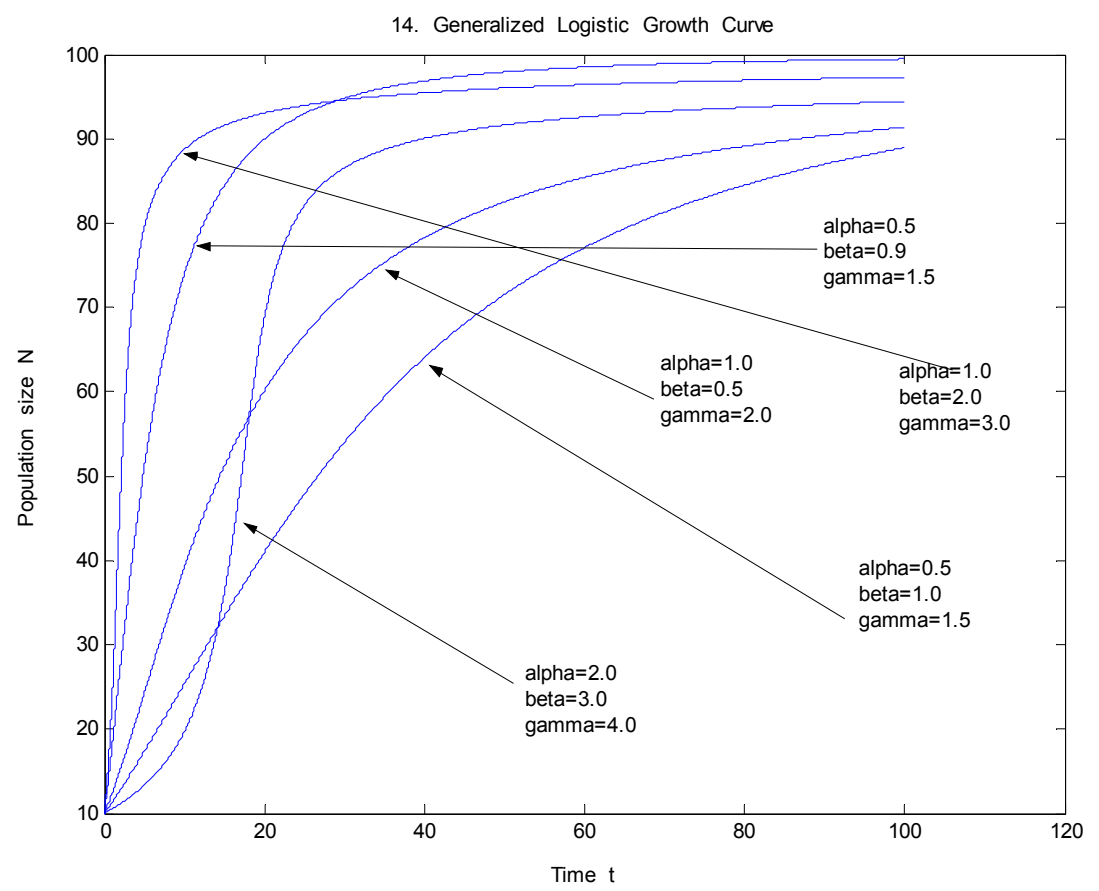

Figure 14. The generalized logistic growth rate curve for several parameter triplets $(\alpha, \beta, \gamma)$ and $X_{0}$ $=10, K=100$.

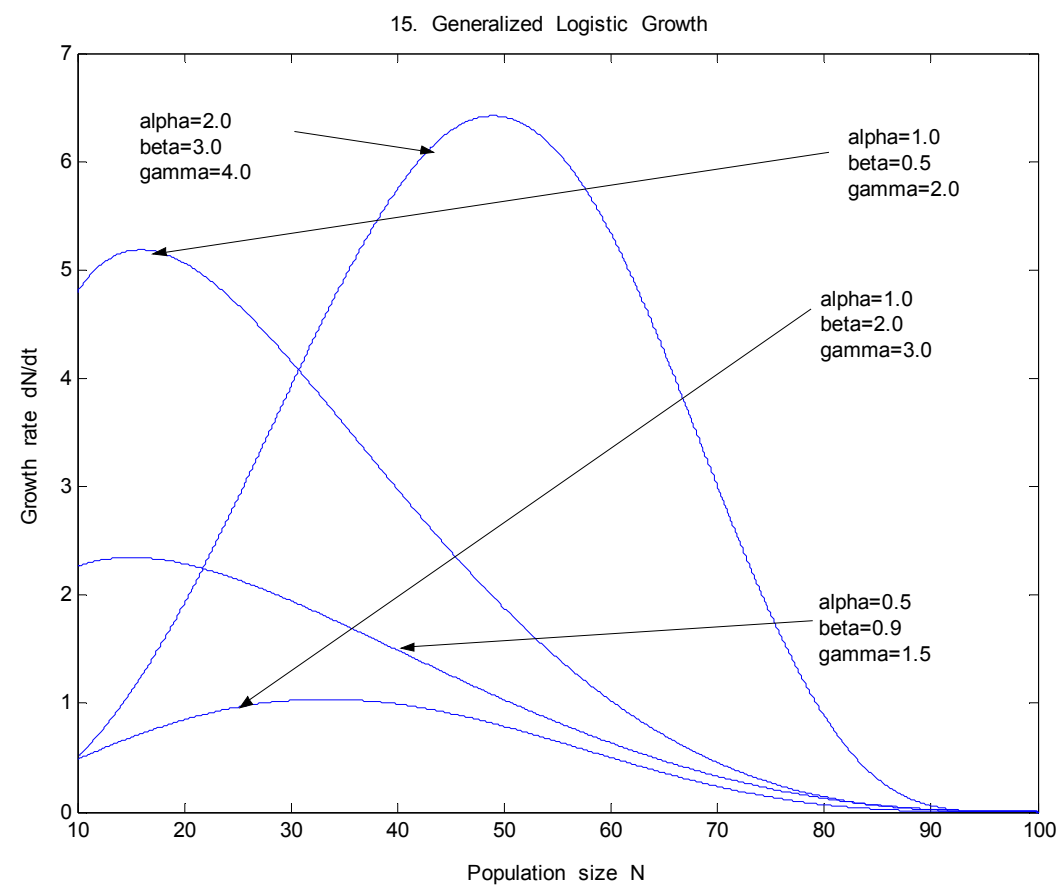

Figure 15. Population growth rate as function of population size according to the generalized logistic form. 
By introducing the auxiliary variable $x=\left(\frac{N}{K}\right)^{\beta}$ we can transform the autonomous differential equation (13) into

$$
\frac{d x}{d t}=\beta r K^{\alpha-1} x^{\frac{\alpha-1}{\beta}+1}(1-x)^{\gamma}
$$

and upon separation of variables and subsequent integration from 0 to $t$

$$
\int_{\left(\frac{N_{0}}{K}\right)^{\beta}}^{\left(\frac{N(t)}{K}\right)^{\beta}} x^{\frac{1-\alpha}{\beta}-1}(1-x)^{-\gamma} d x=\beta r K^{\alpha-1} t
$$

For a certain configuration of parameters the above integral is the Incomplete Beta Function [21]. Specifically, the parameter configuration (assuming again all parameters are positive) is $0<\alpha<1, \beta>0,0<\gamma<1$

By expanding binomially the $(1-x)^{-\gamma}$ term in the integrand of (10) step by step integration can be performed. For notational convenience let $\theta=\frac{1-\alpha}{\beta}-1$. Then

$$
\left[\frac{x^{\theta}}{(\gamma-1) !} \sum_{i=1}^{\infty} \frac{(\gamma+i-2) ! x^{i}}{(i-1) !(\theta+i)}\right]_{x=\left(\frac{N_{0}}{K}\right)^{\beta}}^{x=\left(\frac{N(t)}{K}\right)^{\beta}}=\beta r K^{\alpha-1} t
$$

The above form provides an analytical expression of $t$ as a function of $x=\frac{N}{K}$. An alternative, but equivalent, infinite series expression to (11), taken from Abramowitz and Stegun [22], is reported in the book by Banks [23]. The more desirable functional form, $x(t)=\frac{N(t)}{K}$, is one of the real roots of the polynomial arising from truncating (11) thus,

$$
\sum_{i=1}^{n} a_{i} x^{\theta+i}+g(t)+O\left(x^{\theta+n+1}\right)=0
$$

where $g(t)=-\beta r K^{\alpha-1} t+$ constant and $a_{i}=a_{i}(\gamma, \theta)$, are coefficients functionally dependent on $\gamma$ and $\theta$.

\subsection{Comments on the inflection value $N_{i n f}$}

In order to assess properly the evolution of the inflection value, $N_{i n f}=\left(1+\frac{\beta \gamma}{\alpha}\right)^{-\frac{1}{\beta}} K$, with the simultaneous variation of the parameters $\alpha, \beta, \gamma$ we have created surface plots of $N_{\text {inf. }}$. On each surface plot one parameter is held constant and the other two are allowed to vary.

Figure 16 is a three-dimensional plot of $N_{\text {inf }}$ versus parameters $\beta, \gamma$. Here $\alpha$ as well as $\beta$ and $\gamma$ are allowed to vary continuously in the range $[1.0,100.0]$. It can be seen that $N_{\text {inf }} \uparrow$ as $\alpha \uparrow$. This is because

$$
\frac{\partial N_{i n f}}{\partial \alpha}=\frac{K \gamma}{\alpha^{2}}\left(1+\frac{\beta \gamma}{a}\right)^{-\frac{1}{\beta}-1}>0
$$


For $\alpha=\beta=\gamma=100.0, N_{\text {inf }} \approx K=100$. For $\alpha=\gamma=100.0$ and $\beta=10.0, N_{\text {inf }} \approx 50$, whereas for $\alpha=\beta=100.0$ and $\gamma=10.0, N_{\text {inf }} \approx 100$. For much smaller values of $\alpha$, say $\alpha=$ 10.0 we have a similar situation: for $\beta=100.0, \gamma=10.0, \quad N_{\text {inf }} \approx 100$, and for $\beta=10.0, \gamma=100.0, N_{\text {inf }} \approx 10$. This indicates that for relatively small values of $\beta$, large values of $\gamma$ have a moderating effect on the growth of the inflection value regardless of the value of $\alpha$.

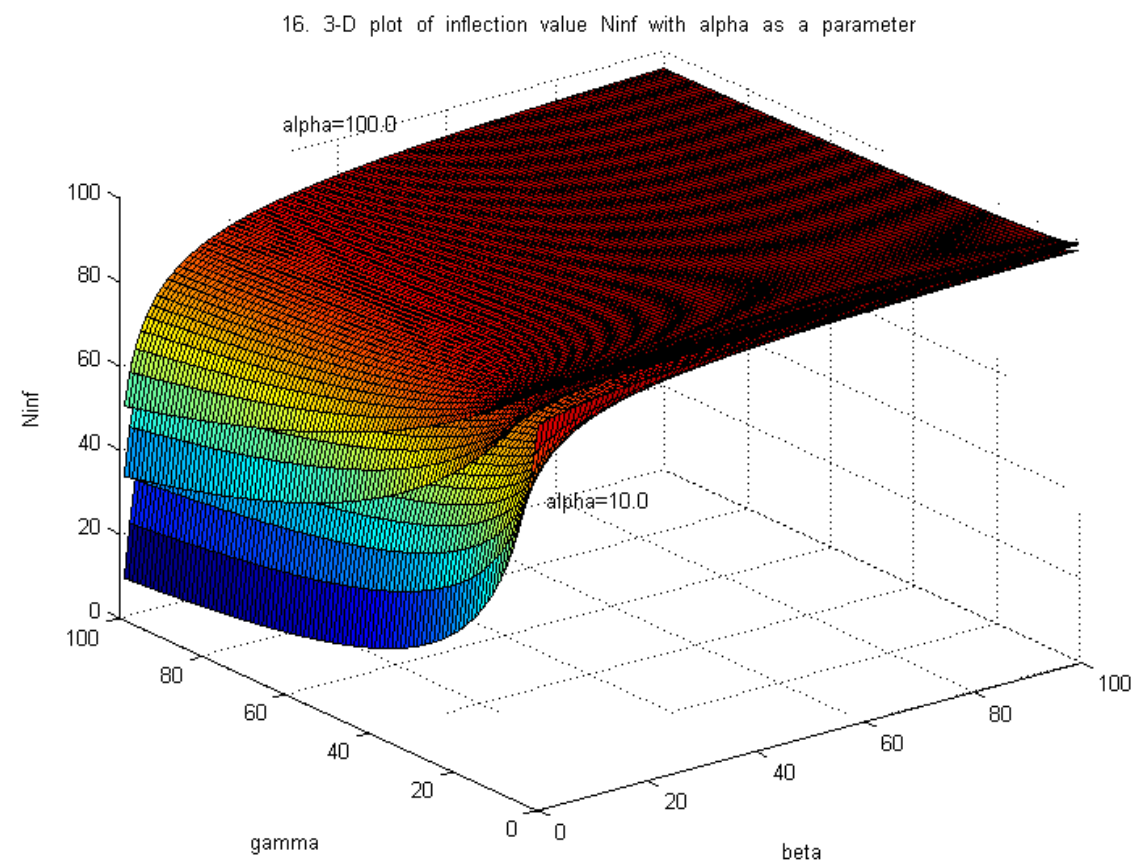

Figure 16. Surface plots of the inflection value, $N_{i n f}(K=100)$ as a function of two variables $\beta, \gamma$. Each surface corresponds to a particular value of the parameter

Figure 17 is a three-dimensional plot of $N_{\text {inf }}$ versus parameters $\alpha, \gamma$. Here again $\alpha, \beta, \gamma$ are allowed to vary continuously in the range [1.0, 100.0]. Again it can be seen that $N_{\text {inf }} \uparrow$ as $\beta \uparrow$. This is because

$$
\frac{\partial N_{i n f}}{\partial \beta}=\frac{K}{\beta}\left(1+\frac{\beta \gamma}{\alpha}\right)^{-\frac{1}{\beta}}\left[\frac{1}{\beta} \ln \left(1+\frac{\beta \gamma}{\alpha}\right)-\frac{\frac{\gamma}{\alpha}}{1+\frac{\beta \gamma}{\alpha}}\right]
$$

$>0$

The resulting inequality is established by observing that the following inequality

$$
\ln y>\frac{y-1}{y} \text { for } y>1
$$

where $y=1+\frac{\beta \gamma}{\alpha}$, is always true given the restrictions on $\alpha, \beta$ and $\gamma$. 
It is obvious from the plot that $\beta$ is the dominant parameter in that large values of $\beta$ result in a high inflection value, $N_{\text {inf }}>90$, regardless of the magnitude of the other two parameter values. For small values of $\beta$ however, say $\beta=0.5, \alpha$ is the dominant parameter in that small values of $\alpha$ will drive the inflection value to zero and large values of $\alpha$ will result in a rapid increase of the inflection value.

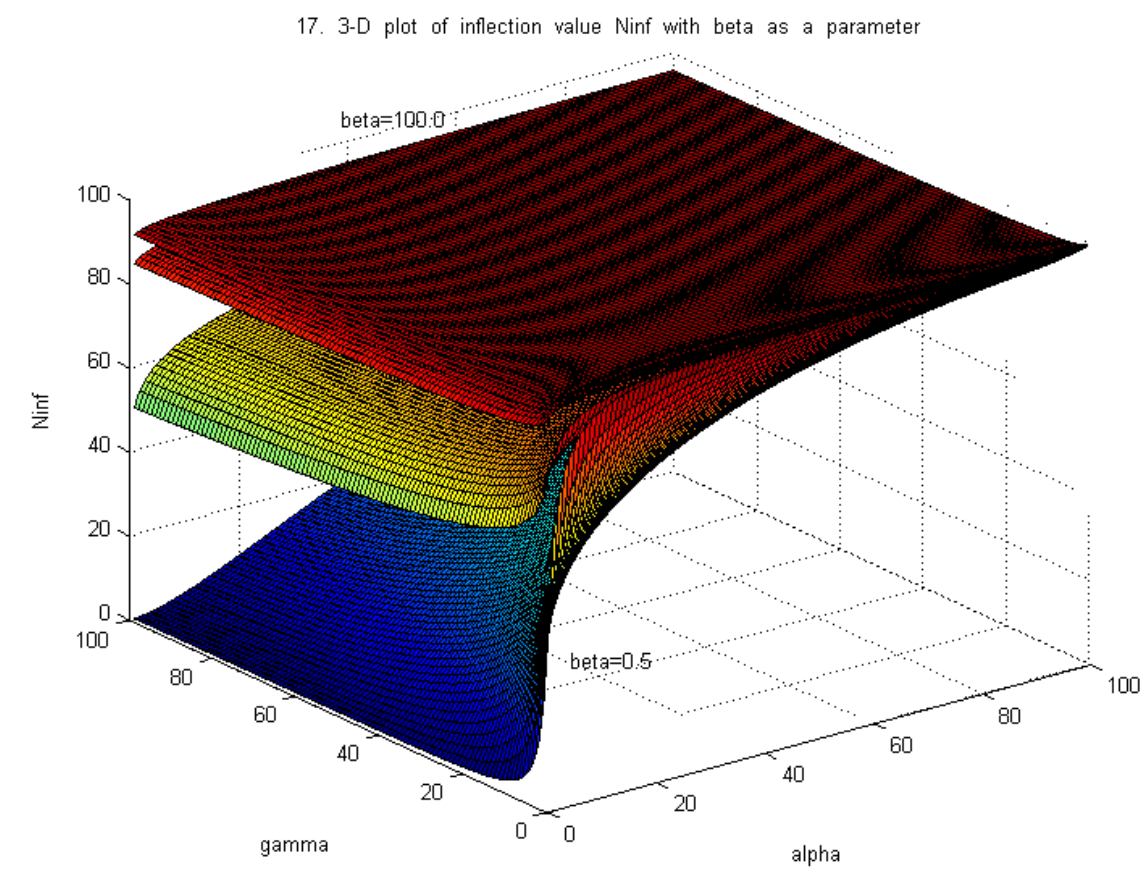

Figure 17. Surface plots of the inflection value, $N_{\text {inf }}(K=100)$ as a function of two variables $\alpha, \gamma$. Each surface corresponds to a particular value of the parameter $\beta$ in the range $[0.50,100.0]$.

Figure 18 is a three-dimensional plot of $N_{i n f}$ versus parameters $\alpha, \beta$. Here also $\alpha, \beta, \gamma$ are allowed to vary continuously in the range $[1.0,100.0]$. In this case however, $N_{\text {inf }} \downarrow$ as $\gamma-$ because

$$
\begin{aligned}
\frac{\partial N_{i n f}}{\partial \gamma} & =-\frac{K}{\alpha}\left(1+\frac{\beta \gamma}{\alpha}\right)^{-\frac{1}{\beta}-1} \\
& <0
\end{aligned}
$$

Very small values of $\alpha$ and $\beta$ rapidly drive the inflection value to zero regardless of the value of $\gamma$. Large values of either $\alpha$ or $\beta$, or both, will restore the inflection value to its asymptotic value when $\gamma$ is relatively small, say $\gamma=10.0$. When $\gamma$ is large however, say $\gamma=100.0$, large values of $\beta$ and small values of $\alpha$ result in a much more rapid growth of the inflection value towards $K$ than correspondingly large values of $\alpha$ and small values of $\beta$, which produce a maximum inflection value of 50 . 


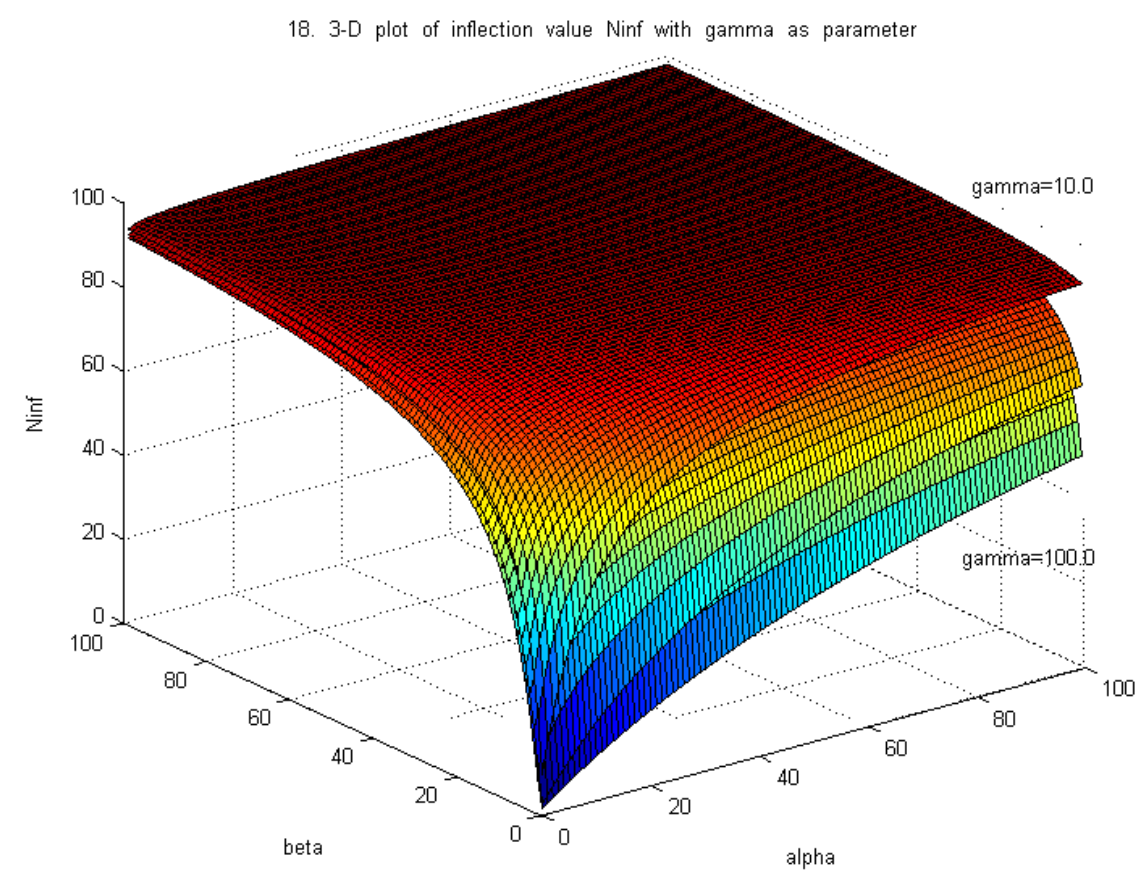

Figure 18. Surface plots of the inflection value, $N_{\text {inf }}(K=100)$ as a function of two variables $\alpha, \beta$. Each surface corresponds to a particular value of the parameter $\gamma$ in the range $[10.0$, 100.0].

It is clearly evident from inspection of figures 16,17 and 18 that for intermediate to large values of $\alpha, \beta, \gamma$ (typically $\alpha>10, \beta>40, \gamma>40$ for figures 16 and 17 and $\alpha>40, \beta>40, \gamma>10$ for figure 18) the corresponding surfaces consistently exhibit approximately zero curvature, that is, they are effectively planes. This is seen immediately from considering the total differential $d N_{\text {inf }}$ along any surface, for example, $\alpha=$ constant (exhibited in figure 16):

$$
d N_{i n f}=\frac{f N_{i n f}}{f \beta} d \beta+\frac{f N_{i n f}}{f \gamma} d \gamma
$$

We are going to prove that $d N_{\text {inf }}$ vanishes identically on the surface $\alpha=$ constant by proving that $\frac{\partial N_{i n f}}{\partial \beta}$ and $\frac{\partial N_{i n f}}{\partial \gamma}$ vanish identically for relatively large values of $\beta$ and $\gamma$. Since $\frac{\beta \gamma}{\alpha} \gg>1$ we shall use the approximation $1+\frac{\beta \gamma}{\alpha} \approx \frac{\beta \gamma}{\alpha}$.

$$
\begin{aligned}
\frac{\partial N_{i n f}}{\partial \gamma}= & -\frac{K}{\alpha}\left(1+\frac{\beta \gamma}{\alpha}\right)^{-\frac{1}{\beta}-1} \approx \\
& -\frac{K}{\alpha}\left(\frac{\beta \gamma}{\alpha}\right)^{-\frac{1}{\beta}-1}= \\
& -K \alpha^{\frac{1}{\beta}}\left(\frac{1}{\beta \gamma}\right)^{\frac{1}{\beta}+1}
\end{aligned}
$$


Then

$$
\lim _{\beta \rightarrow \infty, \gamma \rightarrow \infty} \frac{\partial N_{i n f}}{\partial \gamma}=\lim _{\beta \rightarrow \infty, \gamma \rightarrow \infty}\left[-K \alpha^{\frac{1}{\beta}}\left(\frac{1}{\beta \gamma}\right)^{\frac{1}{\beta}+1}\right]=0
$$

Next consider the limit of $\frac{\partial N_{\text {inf }}}{\partial \beta}$ after rearranging terms:

$$
\begin{aligned}
\frac{\partial N_{i n f}}{\partial \beta}= & \frac{K}{\beta}\left(1+\frac{\beta \gamma}{\alpha}\right)^{-\frac{1}{\beta}}\left[\frac{1}{\beta} \ln \left(1+\frac{\beta \gamma}{\alpha}\right)-\frac{\frac{\gamma}{\alpha}}{1+\frac{\beta \gamma}{\alpha}}\right] \approx \\
& \frac{K}{\beta}\left(\frac{\beta \gamma}{\alpha}\right)^{-\frac{1}{\beta}}\left[\frac{1}{\beta} \ln \left(\frac{\beta \gamma}{\alpha}\right)-\frac{1}{\beta}\right]= \\
& K\left(\frac{\gamma}{\alpha}\right)^{1-\frac{1}{\beta}} \frac{1}{\beta}\left[\frac{\ln \left(\frac{\beta \gamma}{\alpha}\right)}{\frac{\beta \gamma}{\alpha}}-\frac{\alpha}{\beta \gamma}\right]
\end{aligned}
$$

Then

$$
\begin{aligned}
& \lim _{\beta \rightarrow \infty, \gamma \rightarrow \infty} \frac{\partial N_{i n f}}{\partial \beta}= \\
& \lim _{\beta \rightarrow \infty, \gamma \rightarrow \infty} K\left(\frac{\gamma}{\alpha}\right)^{1-\frac{1}{\beta}} \frac{1}{\beta^{1+\frac{1}{\beta}}} \lim _{\beta \rightarrow \infty, \gamma \rightarrow \infty}\left[\frac{\ln \left(\frac{\beta \gamma}{\alpha}\right)}{\frac{\beta \gamma}{\alpha}}-\frac{\alpha}{\beta \gamma}\right]=
\end{aligned}
$$

0

since $\lim _{y \rightarrow \infty} \frac{\ln y}{y}=0$, where $y=\frac{\beta \gamma}{\alpha}$, and $\lim _{\beta \rightarrow \infty, \gamma \rightarrow \infty} \frac{\alpha}{\beta \gamma}=0$.

This directly implies that for an infinite configuration of any two parameters and a fixed (but relatively high) value of the remaining parameter, the time needed by the population (or growing organism) to reach a given inflection value, $t_{\text {inf }}$, is not unique, or equivalently, that a given inflection value, $N_{i n f}$, can be attained via many different parameter values and hence at different times $t_{i n f}$.

\section{Discussion}

The classical Logistic growth equation of Verhulst has been used a basis for several extended models. Each is shown to accommodate population or physical growth without the restriction that the maximum growth rate must, rather artificially, occur at half of the carrying capacity in the case of population dynamics, or maximum attainable dimension for physical growth, for the system being modelled. Properties and restrictions previously unreported for these models are identified and presented. 
A generalized form of logistic growth (13) has been introduced which encompasses extended logistic growth models as special cases. We have shown that the general solution to (13) is an Incomplete Beta Function which has been tabulated by Pearson [21]. In addition we have dealt rigorously, for the first time, with the population at the inflection point, $N_{\text {inf }}$, of all the growth models presented. Furthermore, for this generalised form, we have proved that for extreme values of certain parameters, the population will reach a fraction of the carrying capacity, $K$. This is a novel feature possessed by the generalized logistic growth curve alone and differentiates it from the Verhulst logistic growth curve (1) and the extended forms which have been discussed. It also emphasizes its modelling utility in situations where a biological population (or growing organism) is unable, for whatever reason, to reach the expected carrying capacity of the environment (or limiting dimension).

\section{References}

[1] P.F. Verhulst, Notice sur la loi que la population suit dans son accroissement, Corr. Math. Physics, 10 (1838) 113.

[2] T.Carlson, Über geschwindigkeit und grösse der hefevermehrung in würze, Biochem. Z, 57, (1913), 313-334.

[3] R.Pearl, The growth of populations, Quarterly Review of Biology, 2, (1927), 532-548.

[4] R.Pearl, Introduction of Medical Biometry and Statistics, Saunders, Philadelphia, 1930.

[5] B.J.T. Morgan, Stochastic models of groupings changes, Advances in Applied Probability, 8, (1976), 30-57.

[6] C.J.Krebs, The Experimental Analysis of Distribution and Abundance, Harper and Row, New York, 1985.

[7] T.C.Fisher, R.H.Fry, Tech. Forecasting Soc. Changes, 3, (1971), 75.

[8] C.Marchetti, N.Nakicenovic, The Dynamics of Energy Systems and the Logistic Substitution Model, Int. Inst. for Appl. Sys. Anal., Laxenburg, Austria, 1980.

[9] R.Herman, E.W.Montroll, Proceedings of the National Academy of Sciences, USA, 69, (1972), 3019.

[10] A.J.Lotka, Elements of Mathematical Biology, DOVER, New York, 1956.

[11] R.Pearl, L.J.Reed, On the rate of growth of the population of United States since 1790 and its mathematical representation, Proceedings National Academy of Sciences USA, G (1920), 275.

[12] F.J.Richards, A Flexible Growth Function for Empirical Use, Journal of Experimental Botany, 10(29), (1959), 290-300.

[13] L.Von.Bertalanffy, "A quantitative theory of organic growth, Human Biology, 10(2), (1938), 181-213.

[14] J.A.Nelder, The fitting of a generalization of the logistic curve, Biometrics, 17, (1961), 89-110.

[15] A.A.Blumberg, Logistic Growth Rate Functions, Journal of Theoretical Biology, 21, (1968), 42-44.

[16] M.E.Turner, B.A.Blumenstein , J.L.Sebaugh, A generalization of the logistic law of growth, Biometrics, 25, (1969), 577-580.

[17] M.E.Turner, E.Bradley, K.Kirk, K.Pruitt, A Theory of Growth, Mathematical Biosciences, 29, (1976), 367-373.

[18] R.Buis, On the Generalization of the Logistic Law of Growth, Acta Biotheoretica, 39, (1991), 185-195.

[19] J.W.Haefner, Modeling Biological Systems, ITP, 1996.

[20] H.McCallum, Population Parameters: Estimation for Ecological Models, Blackwell Science, UK 2000.

[21] K.Pearson, Tables of the Incomplete Beta Function, Library Binding, Lubrecht and Cramer Ltd., 1968. 
[22] M.Abramowitz, I.M.Stegun, Handbook of Mathematical Functions, Dover, New York, 1965.

[23] R.B.Banks, Growth and Diffusion Phenomena, Springer Verlag, 1994. 